\title{
A Survey of Traffic Sign Recognition
}

\author{
Ling Zhong \\ Shen Yang University of Technology \\ school of software \\ Shen Yang, LiaoNing, China \\ e-mail: xuzhongzh@sina.com
}

\section{Yajie $\mathrm{Yu}$ *}

Shen Yang University of Technology

school of information science and Engineering

Shen Yang, LiaoNing,China

e-mail: yuyajie0501@163.com

* Corresponding Author

\author{
Zhijia Zhang \\ Shen Yang University of Technology \\ school of software \\ Shen Yang, LiaoNing, China \\ e-mail: 429239249@qq.com
}

\begin{abstract}
As the main component of intelligent transportation systems, Traffic sign recognition system has been extensively studied in intelligent vehicles, military and unmanned vehicles and other projects. In this paper, firstly, it briefly introduced the traffic sign recognition research status and development background, and then according to different recognition review, it mainstreamd traffic sign recognition method, through comparative analysis of various methods, it summarized the in traffic sign recognition, and prospected the future direction of development.
\end{abstract}

Keywords- Traffic sign recognition; intelligent transportation systems; traffic signs;SVM; Neural Networks

\section{INTRODUCTION}

In recent years, many countries have carried out research on intelligent driving system. Intelligent driving set communication, detection, control and computer technology in one, and by automatically identifying traffic information, giving driving tips, so it can realize a semiautomated, unmanned driving.

As the important part of intelligent driving system, Traffic sign recognition (Traffic Sign Recognition, TSR) is a typical application of image processing, pattern recognition, machine vision and other multidisciplinary research, as well as the difficulty of real pattern recognition, one of the unresolved problems in the research field of intelligent driving system. Therefore, TSR has become a hot topic in domestic and foreign academic research and industry application in recent years.

\section{METHODS AND DEVELOPMENT OF TRAFFIC SIGN RECOGNITION}

Traffic sign recognition algorithm is various. To enhance the recognition efficiency and effect, it has been gradually showing a different algorithm combining research trends. Here are several commonly used methods of traffic sign recognition.

\section{A. Traffic Sign Recognition Method Based on Statistical Classification}

The basic idea of statistical classification method is to use all kinds of distribution characteristics, namely the direct using of various types of probability density function, the posteriori probability, or implicitly using the above concept of classification. Such of theories are more perfect, and there are a lot of methods.

\section{1) Nearest-Neighbor Algorithm}

Nearest neighbor algorithm (KNN algorithm, also translated K- nearest neighbor algorithm)is in the feature space, depending on the distance between the training samples to identify the mode. The cases have high similarity to each other which are the same category.By calculating the similarity between known cases, it can evaluate the unknown category of cases.

P. Sompoch, who used the nearest neighbor method ${ }^{[1]}$, classified traffic sign according to the Euclidean distance between the samples and standard templates. H.Fleyeh and E.Davami extracted the most effective feature vector from the samples by principal component analysis $(\mathrm{PCA})^{[2]}$, and calculated a set of weights, using Euclidean distance to classify unknown samples.

Bacause the distance measurement can not overcome the correlation between vectors, it can result in the classification results with a high chance.

\section{2) Correlation Coefficient Algorithm}

The similarity coefficient is a measure index of the degree of similarity between two categories. Samples' properties is closer, and the absolute value of the similarity coefficient is closer to 1 , while the absolute value is closer to 0 .

In 1995, M.Betke et al.through similar traffic signs coefficient between images and standard templates, achieved the classification of traffic signs ${ }^{[3]}$. The similarity coefficient is greater, indicating the similarity probability of traffic signs images and standard template is greater.

3) Clustering Analysis Method

Cluster analysis method is that the similar objects through a static classification, are divided into different 
groups or subsets, so that the members of the same subset have similar properties.

Such as the United States, using color clustering method on the "stop" sign, developed ADIS system ${ }^{[4]}$ with the correct identification of all the samples,but the system was not a real-time processing system taking a long time.

4) Decision Tree Classification Method

A decision tree is a predictive model, which represents a mapping relationship between object attributes and object values. Each node in the tree represents an object, and each bifurcated path represents a possible attribute values, and each leaf node corresponds to the path from the root node to the leaf node experiences represent value.

The decision tree classification of multilayer structure can accelerate the processing speed, improve the classification accuracy, and simplify the design by decomposing feature information to each layer. Therefore, some scholars have proposed a multi-layer decision tree classification methods, such as W.Ritter proposed a three layers classification decision tree according to color, shape and primitive ${ }^{[5]}$. P. Pacllk classified the samples according to the color characteristics and geometry characteristics ${ }^{[5]}$. At present, it is hard that optimizing the design and selecting the parameter. and mainly rely on empirical knowledge.

\section{B. Traffic Sign Recognition Method Based on Template Matching}

Template matching is that establishing a template library, in which are standard traffic sign images as templates, comparing a sample and each template, directly calculating the matching degree of two images, finding out the most similar standard template. If the similarity degree does not exceed the predetermined value, the sample will belong to the standard template category.

With the in-depth study of template matching technology, there are some commonly used methods:

1) Matching Based on Pixel Level

It mainly uses the point matching, region matching, and achieves the matching of pixel gray level based on the original image after smoothing. The advantage is convenient way, simple matching algorithm; and the deficiency is computationally intensive, real-time differential.

In 1987, Japanese scholar Akastuka recognized the speed limit traffic signs ${ }^{[6]}$, using threshold segmentation, template matching method and hardware implementation. Piccioli extracted the traffic signs interior design, matching with the database temple, but this method does not consider the effect of light, so it can be used only in laboratory. It is difficult to adapt to the actual reality in the environment recognition. In 2000, Japan Osaka University developed a active vision system that can recognize speed sign and text ${ }^{[7]}$, detecting by threshold segmentation, recognizing using template matching method.

\section{2) Matching Based on Feature Class}

It is extracting an effective feature quantity from the original image to match. This method is more flexible with a small amount of computation, and image processing personnel have more space to play. But it is difficult to find a unified feature extraction criteria, usually can only be confirmed by experiment.
Wang Yang of Jilin University, adopted a template matching method based on moment invariant features ${ }^{[8]}$, to improve the recognition rate, and optimize recognition performance. Piccioli of Italy, proposed a template matching method based on geometric information [9], which had a good recognition rate, but the speed is slow. Fang Ze ping of Beijing University of Technology, using a template matching method ${ }^{[10]}$ based on color feature. By using the statistical characteristics of image pixel, he calculated the matching degree, to realize the traffic signs recognition with good accuracy. In 1996, Jiang Gang yi, Zheng Yi using mathematical morphology method and morphological skeleton function as template matching ${ }^{[11]}$ characteristics for warning signs, got better recognition of traffic signs, and shift invariance and robustness of recognition.

\section{(3) Matching Projection Method}

It uses the matching projection on the test sample, and finds a group of primitives on behalf of signs, then each sign can be described by the corresponding coefficient vector. Take this set of primitives to classify the input images, and do not need to compare input images with all templates, because it can be only matched with templates combined by primitives, so it can improve its processing speed. As S.H.Hsu et al, proposed classification method of projection $^{[5]}$, this kind of method has the disadvantages of poor resistance, large amount of calculation.

\section{Traffic Sign Recognition Method Based on Syntactic Classification}

For complex target, feature vector combined by numerical characteristics can not reflect the configuration information of target. Therefore, the syntactic pattern recognition method of description and recognition of target by using characteristics of the object, is also the focus point in traffic sign recognition.B.Bessere et al., according to colors, shapes, basic primitives and the mutual position ${ }^{[5]}$, described traffic signs by building the semantic web. For the uncertain knowledge, it uses evidence theory fusion method for classification of traffic signs.At present, the computer representation, semantic network composition and structure analysis, algorithm of the realtime aspects traffic signs on prior knowledge need to be further improved.

\section{Traffic Sign Recognition Method Based on Machine Learning}

1) Support Vector Machine

Support vector machine (SVM), a optimal classification model based on database, has the advantages of simple structure, strong generalization ability and other excellent properties. It has a outstanding performance in solving small sample, nonlinear and high dimensional space pattern recognition problem, and it has become a new bright spot in machine learning.

In 2007, Japan developed an automatic detection and recognition system based on vector machines ${ }^{[12]}$.It can detect circular, rectangular, triangular, regular octagon, and color segmentation on HIS color space. In 2010, Spain's Makionadoli et al. using SVM method, the recognition rate reached $95.5 \%{ }^{[13]}$. Wang Nan form Northeastern University, proposed a traffic sign detection and tracking method based on multi-threaded mixed ${ }^{[14]}$ in 2010 , the algorithm, which extracting wavelet features, using two 
binary tree support vector machine classification, had a better recognition accuracy, higher time complexity.

However, the support vector machine (SVM) is only suitable for linear separable case, and the processing time is longer. In order to meet the requirement of real-time traffic sign recognition, the algorithm should be further improved.

\section{2) Neural Networks}

Neural network can automatically extract the sample characteristics, access rules and features of traffic signs images while other methods is difficult to achieve implicit representation, avoid the complexity of feature extraction work. This method is conducive to the realization of the hardware and can handle some of the more complex information, background knowledge is unclear, and allows the target sample a larger defect, distortion, with an adaptive fault-tolerant, strong learning ability, quick recognition speed.

In 1992, French Saint-Blancard developed a red sign recognition system ${ }^{[15]}$, using the color (red) filtering, edge detection and closed curve of edge detection method, and neural network for target classification. Kellmeyer et al, used a neural network with color segmentation and shape analysis algorithm ${ }^{[16]}$, but the rate of localization and recognition is in general. Spain A.delaEscalera proposed a genetic algorithm and neural network traffic sign positioning ${ }^{[17]}$ under complex situation recognition, but the recognition rate is high, while speed is in general.In 2000, Taiwan, China, began research in this area. National Chengchi University ${ }^{[18]}$, National Taiwan Normal University, ${ }^{[19]}$, etc. had published many articles about the traffic sign detection and recognition of academic papers. The detection was still dominated by features such as color and shape, classification approach was neural network. In 2001, Liu and Ran of University of Wisconsin in the United States, identified stop signs with ${ }^{[20]}$ neural network, color segmentation in HSI space, but did not explain the processing time and real-time. In 2002, American achieved traffic sign detection and recognition with HSV space dynamic threshold and Multilayer Perceptron Neural Network [21], which speed and recognition rate were superb.In 2004, Wang Kunming, who used four layers BP neural network classification methods ${ }^{[22]}$, improved the training speed and recognition accuracy, but only for a limited number of signs. In 2007, Moutarde et al regarded Europen and United States speed limit signs as the goal, recognition by ${ }^{[23]}$ neural network method. In 2012, Dan Ciresan and Ueli Meier when attending The German Traffic Sign Recognition Benchmark game, proposed a ${ }^{[24]}$ Multi-Column Deep Neural Network method. The method combined some neural networks trained on a variety of different pretreatment, and further improved the recognition performance and generalization ability.

\section{E. Traffic Sign Recognition Method Based on Integrated Classification}

In order to improve the recognition effect, there are also scholars integrating method.

V.Rehrmatin et al, proposed a method based on combining neural networks and nearest neighbor method to classify the traffic signs ${ }^{[25]}$, which has strong robustness in natural outdoor scene conditions, and it can reach $98 \%$ recognition rate. Although the recognition rate is higher, but such integration methods in structural analysis, realtime system and other aspects of the algorithm still needs to be relatively large improvements.

\section{The DifFICUlties In TRAFFIC SIGN RECOGNITION}

The existing recognition algorithms in a restricted conditions (controlled light, normal shape, etc.) achieve satisfactory performance, but they can not meet the requirements that high recognition rate in the objective environment, especially with occlusion, noise, illumination variety, shape distortion and other serious challenges in many aspects. Factors affecting the traffic sign recognition are the following main aspects:

\section{A. Change of Light}

Changes in the light environment can cause changes of traffic signs light reflective, so that the brightness and contrast of the collected samples change. In addition, traffic signs can reflect light at night, which will distortion the original color.

\section{B. Change in Shape}

Different focal length of different imaging devices and different imaging distance will result in the different sample size.

\section{C. the Impact of Occlusion}

Trees, vehicle and other obstructions can block the traffic sign regional, which will significantly increase the difficulty of two-dimensional traffic sign image identification.

\section{D. the Huge Amount of Image Data}

Since the sample is two-dimensional or color, an enormous amount of data will be in the system, consuming a lot of resources and time.

\section{E. Insufficient Recognition Speed and Poor Scalability}

The existing algorithms deficient recognition speed, extended inconvenience etc.For many better recognition rate algorithms, response time is often longer, particularly difficult to achieve in an embedded device.

\section{F. Improvement of the Generalization Ability}

Traffic signs in China is not exactly the same with other countries, and it can not only our traffic signs of generalization to other traffic signs.

\section{CONCLUSIONSY AND PROSPECT}

After decades of research, traffic sign recognition has made great progress, but because the existing algorithm can solve the single problem, the realization of universal traffic sign recognition system is still the main research topics.Successful traffic sign recognition systems need to consider the following aspects: (1)Research of preprocessing algorithm. For some of the existing recognition algorithms, the preprocessing is used relatively simple methods, such as color space transformation, size adjustment, etc. Preprocessing algorithm should be able to suppress interference to lay a better foundation for identification. (2)Research of identification algorithm. Experimental samples are collected under natural 
conditions, so there must be distorted, obscured, discoloration, etc. The algorithm should not only have enough capacity ability, but also can not ignore the effective feature. (3) Improvement of the recognition speed The requirement of real-time algorithm is the precondition of the application of the algorithm, and traffic sign recognition algorithm is required a high degree of real-time. It will mark the road to collect real-time processing, and recognition results will be timely feedback, so that the traffic sign recognition system could be applied to intelligent vehicles and unmanned vehicle. (4) the video image processing. By placing the camera in a moving vehicle, he road traffic signs to be collected, then the algorithm requires to realize the detection and recognition of video image.

As the traffic sign recognition theory is not perfect, the specific algorithm implementation are more to be studied, so the traffic sign recognition research is still require researchers to make unremitting efforts.

\section{ACKNOWLEDGMENT}

Project Name: Research on key technology of fire warning indoor public place based on multi source detection

Liaoning Province Outstanding Talent Cultivation Plan Project (2014020116)

\section{REFERENCES}

[1] P.Sompoch,C.XiaoYang,K.Michiro.Automatic recognition and location of road signs from terrestrial color imagery. Bangkok,Thailad, Geoinformatics\& DMGIS'2001,2001:pp.238247.

[2] H.Fleyeh,E.davami. Eigen-based traffic sign recognition. IET Intelligent Transport Systems,2011,Vol.5(3): pp.190- 196.

[3] M.betke,N.C.Makris.Fast object recognition in noisy images using simulated annealing. Cambridge, USA,Proceedings of the IEEE International Conference on Computer Vision,1995:pp.523-530.
[4] N.Kehtarnavaz,N.C.Grisworld,D.S.Kang.Stop-sign recognition based on color shape processing[J].Machine Vision and Applications, 1993,6:pp.206-208.

[5] H.Akatsuka,S.Imai. Road signposts recognition system . SAE transactions, 1987, Vol.96, pp.936-943..

[6] Jun Miura,Tsuyopshi Kanda,Yoshiaki Shirai.An Active Vision System for Real-time Traffic Sign Recognition. Dearborn ML.USA:IEEE on Intelligent Transportation System,2000,1748:52-57.

[7] G.Piccioli,E.D.Michel,P.Pandi,M.Campani.Robust method for road sign detection and recognition .Image and vision computing,1996, Vol.14(3),pp.119-129.

[8] Mal donado Bascon.Road-Sign Detection and Recognition Based on Support Vector Machines . Intelligent Transportation Systems,2007, Vol8(2): pp.264-278.

[9] Maldonado Bascon,S.Acevedo Rodriguez, J.Lafuente .An optimization on pictogram identification for the road-sign recognition task using SVMs. Computer Vision and Image Understanding,2010,Vol.114(3),pp.373-383

[10] M.de Saunt Blancard. road sign recognition :A study of visionbased decision making for road environment recognition. Visionbased Vehicle Guidance,Springer Series in Perception Engineering, 1992.

[11] D.Kellmeyer,H.Zwahlen.Detection of Highway Warning Signs in Natural Video Images Using Color Image Processing and Netural and Networks. IEEE,0-8186-6250-6/94,June1994:pp.88-93

[12] A.de la Escalera, J.M.A Armingol, M.Mata.Traffic sign recognition and analysis for intelligent vechiles. Image Vision Computer,1994,Vol.21:pp.247-258.

[13] X.Herry,Bin Ran.Vision-Based Stop Sign Detection and Recognition System for Intelligent Vehicle. Transportation Research Record,2001:pp.161-166

[14] S.Vitable,A.Gentile,F.Sorbello.A neural network based automatic road signs recognizer. Honolulu,America:the 2002 International Joint Conference on Neural Networks,2002:315-320.

[15] D. C. Ciresan, U. Meier, L .M. Gambardella , and J . Schmidhuber. Convolutional neural network committees for handwritten character classification. In International Conference on Document Analysis and Recognition,2011.Vol.1(3),pp.1250-125

[16] V.Rehrmann,R.Lakmann,L.Priese.A Parallel System for Real-time Traffic Sign Recognition. Peking, China, Proceedings International Workshop on Advanced Parallel Processing Technologies95, 1995:72-78 